\title{
Postoperative analgesia after combined
} thoracoscopic-laparoscopic esophagectomy: a randomized comparison of continuous infusion and intermittent bolus thoracic epidural regimens

This article was published in the following Dove Medical Press journal:

Journal of Pain Research

\author{
Ke Wei \\ Su Min \\ Yonggang Hao \\ Wei Ran \\ Feng Lv \\ Department of Anesthesiology, The \\ First Affiliated Hospital of Chongqing \\ Medical University, Chongqing, \\ People's Republic of China
}

\begin{abstract}
Purpose: Judicious postoperative pain management after thoracoscopic-laparoscopic esophagectomy (TLE) facilitates enhanced rehabilitation. Thoracic epidural analgesia (TEA) offers many benefits in esophagectomy, while several complications are associated with the delivery mode by continuous epidural infusion. This study compared the efficiency and safety of intermittent epidural bolus to continuous epidural infusion for pain management after TLE.
\end{abstract}

Patients and methods: Sixty patients, aged 18-80 years, with American Society of Anesthesiologists classes I-III and scheduled for TLE with combined general anesthesia and TEA were randomly allocated to two groups. Patients received either a continuous epidural infusion with $0.3 \%$ ropivacaine and $1.5 \mu \mathrm{g} / \mathrm{mL}$ fentanyl at $6 \mathrm{~mL} / \mathrm{h}$ plus a patient-controlled bolus of 3 $\mathrm{mL}$ (continuous group) or an intermittent bolus of $6 \mathrm{~mL}$ of the same solution on demand with lockout time of 30 minutes (intermittent group). If the patient complained of pain and the visual analog scale score was $>4$, an intravenous injection of tramadol or dezocine was administered as rescue treatment. The primary outcome variable was the consumption of epidural opioids and local anesthetics for TEA.

Results: TEA for pain management following TLE by intermittent epidural bolus was associated with significantly lower consumption of fentanyl and ropivacaine and lower incidences of breakthrough pain and hypotension than continuous epidural infusion. No significant differences were observed between the two groups in terms of pain score at rest or while coughing, patient satisfaction, or incidence of postoperative complications.

Conclusion: Compared with continuous epidural infusion, TEA by on-demand intermittent bolus greatly reduced the consumption of local anesthetics and opioids with comparable pain relief and little impairment in hemodynamics when used for pain management after TLE.

Keywords: thoracic epidural analgesia, thoracoscopic-laparoscopic esophagectomy, postoperative pain

\section{Introduction}

Esophagectomy remains one of the highest risk thoracic surgical procedures with perioperative mortality rate of $\sim 3 \%$ and major morbidity rates of as much as $30 \%{ }^{1}$ Postoperative pain after esophagectomy can be difficult to manage. Abdominal and thoracic components of the surgical procedure cause protracted wound and visceral pain. In recent years, thoracoscopic-laparoscopic esophagectomy (TLE) has become increasingly popular as a minimally invasive procedure resulting in fewer pulmonary complications, less blood loss, and shorter hospital stays. ${ }^{2,3}$ In this surgical approach,
Department of Anesthesiology, The First Affiliated Hospital of Chongqing Medical University, I Youyi Road, Yuzhong District, Chongqing 4000I6, People's Republic of China

Tel/fax +8623890I 1062

Email wk202448@hospital-cqmu.com 
the pain directly from the surgical incision is greatly reduced, whereas the visceral pain and the thoracic drainage catheters all add to the range of dermatomes for which analgesia needs to be considered.

Thoracic epidural analgesia (TEA) has long been regarded as the mainstay of multimodal analgesia for thoracic and abdominal surgeries. Due to several contraindications and side effects, a series of potential alternative modalities to TEA (intercostal nerve blocks, paravertebral or transverse abdominis plane blockade, and continuous wound catheter infusion of local anesthetic) has been proposed, especially for patients undergoing minimally invasive surgery. However, existing evidence is far from reaching a consensus that the benefits of a sensibly conducted and prolonged epidural analgesia can be completely replaced by one of these techniques alone. ${ }^{4,5}$ In light of its reliable analgesic effect, controllable analgesic range and long duration of the sensory block, TEA is still commonly utilized in open esophagectomy and in TLE by multiple institutions. ${ }^{6}$ Furthermore, TEA as the core component of perioperative analgesia in minimally invasive esophagectomy was highly recommended in the latest enhanced recovery programs for patients undergoing esophagectomy. ${ }^{7}$

In previous studies on labor analgesia or cancer pain management, epidural bolus has exhibited certain advantages over continuous epidural infusion for pain relief. ${ }^{8-11}$ For esophagectomy in which multiple sources of postoperative pain existed, we hypothesized that TEA administered by intermittent bolus could also lead to better outcomes for pain relief. The purpose of the present study was to compare the efficiency and safety of the two epidural regimens for pain management following TLE.

\section{Patients and methods}

The study was approved by the local ethics committee of the First Affiliated Hospital of Chongqing Medical University (Chongqing, People's Republic of China). Written informed consent was obtained from each patient before enrolling in the study. All of the procedures were performed in accordance with the Declaration of Helsinki.

\section{Enrollment}

Sixty patients with American Society of Anesthesiologists classes I-III aged 18-80 years who were scheduled for TLE between October 1, 2017, and May 5, 2018, were enrolled in this prospective, randomized, double-blind study. Patients were excluded from this study if they met at least one of the following criteria: 1) refused epidural analgesia; 2) preopera- tively diagnosed opioid tolerance or chronic pain or a history of thoracic epidural anesthesia/analgesia; 3) contraindications to the epidural analgesia, such as coagulation disorders or infection at the puncture site; 4) morbid obesity, pulmonary infection, or active asthma; 5) inability to comprehend or complete verbal and physical instructions; and 6) required prolonged ( $>2$ hours) mechanical ventilation after surgery.

\section{Monitoring and thoracic epidural catheterization}

All patients were premedicated with $5 \mathrm{mg}$ dexamethasone intravenously 1 hour before induction of anesthesia. Upon arrival in the operating room, patients were monitored with electrocardiography, pulse oximetry, invasive blood pressure, and body temperature. Epidural puncture was performed by experienced anesthetists who were blinded to the study protocol. The epidural catheter was kept at the level of T7/8 interspace for 3-4 cm. A test dose of $3 \mathrm{~mL}, 1.5 \%$ lidocaine with 1:200,000 epinephrine was used to rule out intrathecal or intravascular catheterization (positive response: an increase in heart rate by 15 beats/minute within 45 seconds or a higher block level than expected after 5 minutes). In all patients, 20 minutes after the first epidural injection of 7-10 mL $0.5 \%$ ropivacaine, the sensory block was tested by pinpricks to confirm the analgesic area from T4 to T10 dermatomes. If the required block area was not achieved, a repeated epidural bolus with half of the initial dose of the same solution was added. Patients who did not reach the target block level were excluded from the study.

\section{Intraoperative treatment}

After the epidural block, patients were randomly allocated to the continuous group or the intermittent group using sealed envelopes with computer-generated numbers. General anesthesia was induced with $2 \mathrm{mg} / \mathrm{kg}$ propofol, $0.5 \mu \mathrm{g} / \mathrm{kg}$ sufentanil, $100 \mathrm{mg}$ lidocaine, and $1 \mathrm{mg} / \mathrm{kg}$ rocuronium. A double-lumen tube was intubated into the trachea, and fiberoptic bronchoscopy was used to determine the correct placement of the tube. General anesthesia was maintained with sevoflurane and intravenous infusion of remifentanil $0.08-0.15 \mu \mathrm{g} / \mathrm{kg} /$ minute to maintain Narcotrend values between 40 and 60 . Rocuronium bolus $(0.3 \mathrm{mg} / \mathrm{kg})$ was added as evidenced by lack of train-offour responses to neuromuscular stimulation. Omeprazole (40 $\mathrm{mg}$ ) and tropisetron (5 mg) was intravenously administered at the beginning of the surgery. Mechanical ventilation was maintained at $8 \mathrm{~mL} / \mathrm{kg}$ tidal volume during two lung ventilations or $6 \mathrm{~mL} / \mathrm{kg}$ tidal volume and $5 \mathrm{~cm} \mathrm{H}_{2} \mathrm{O}$ positive end expiratory pressure (PEEP) during one lung ventilation. The 
respiratory rate was adjusted to keep end tidal carbon dioxide pressure between 35 and $45 \mathrm{mmHg}$ throughout the anesthesia. Intraoperative fluid administration was maintained with warmed lactated Ringers solution at $8 \mathrm{~mL} / \mathrm{kg} /$ hour. Blood loss was replaced with allogenic blood if the hemoglobin concentration was lower than $8 \mathrm{~g} / \mathrm{dL}$. Hypotension (>20\% decrease from baseline blood pressure lasting more than 1 minute) was treated with $250 \mathrm{~mL} \mathrm{6 \%}$ hydroxyethyl starch 130/0.4 infusion over 15 minutes. If the hypotension was not corrected, intravenous ephedrine $(5 \mathrm{mg})$ or phenylephrine $(100 \mu \mathrm{g})$ was used. Bladder temperature was maintained between $36^{\circ} \mathrm{C}$ and $37^{\circ} \mathrm{C}$ with the use of a warming system (Cocoon CWS 4000; Care Essentials Pty Ltd, Australia). Arterial blood gas, serum electrolytes, and blood glucose levels were analyzed (GEM premier 3000; Instrumentation Laboratory Company, Bedford, MA, USA) once every hour, and the values were maintained within normal ranges by appropriate treatments.

Surgeries were completed by the same thoracic surgical team who were blinded to the study protocol. The first stage of the surgery consisted of a right video-assisted thoracoscopic surgery with the placement of four thoracoscopic ports. In the laparoscopic approach, $\mathrm{CO}_{2}$ pneumoperitoneum at $12-15 \mathrm{mmHg}$ was maintained. Before closing the cervical incision for anastomosis, all patients were given a loading dose of $6 \mathrm{~mL} 0.3 \%$ ropivacaine with $10 \mu \mathrm{g}$ fentanyl through the epidural catheter. Parecoxib (40 mg) was administered intravenously and repeated every 12 hours.

\section{Blinding and postoperative treatment}

At the end of the surgery, patient-controlled epidural analgesia (PCEA) was started either by continuous infusion (continuous group) or intermittent injections (intermittent group) of $0.3 \%$ ropivacaine and $1.5 \mu \mathrm{g} / \mathrm{mL}$ fentanyl using the same device. Patients were unaware of the epidural regimen, which was set according to the group assignment by anesthetists who were uninvolved in this study. Postoperative assessment was performed by an investigator who was blinded to the patients' group. For the continuous group, a basal continuous epidural infusion $(6 \mathrm{~mL} /$ hour $)$ plus patientcontrolled additional epidural bolus $(3 \mathrm{~mL}$; lockout time, 15 minutes) was administered. For the intermittent group, patients received an intermittent bolus $(6 \mathrm{~mL}$; lockout time, 30 minutes) of the same solution on demand. Postoperative pain level was assessed by visual analog scale (VAS). For both groups, tramadol or dezocine was available as a rescue drug for breakthrough pain (VAS >4) or unsatisfactory pain relief. Treatment of hypotension was consistent with the intraoperative principles. Levels of postoperative pain, consumption of ropivacaine and fentanyl, treatment of breakthrough pain, and incidence of postoperative complications were recorded. PCEA was used for 3 days after surgery, and the epidural catheter was removed on the fourth postoperative day. Residue pain relief was continued with systemic analgesia by intravenous parecoxib.

After the surgery, all patients were immediately transferred to the intensive care unit (ICU). Patients were discharged from the ICU if they met the following discharge criteria: stable hemodynamics without continuous blood loss, normal respiration without hypoxemia under room air, and VAS scores $\leq 4$

\section{Endpoints and sample size}

The efficiency of pain control was assessed by the consumption of epidural drugs, quality of analgesia (in terms of VAS), and incidence of breakthrough pain. The primary outcome was the total consumption of fentanyl and ropivacaine for PCEA. Secondary outcomes included postoperative rehabilitation and adverse effects associated with epidural administration (postdural puncture headache, hypotension, sedation, respiratory depression, nausea, and vomiting). Based on the findings from our pilot study, a sample size of 23 patients per group was estimated with a power of $80 \%$ and a two-sided $P$-value of 0.05 to detect a $80 \mu \mathrm{g}$ difference ( $\sim 20 \%$ reduction) in total fentanyl consumption with comparable pain scores. Considering patients who may withdraw from the study, 30 patients were enrolled in each group.

\section{Statistical analyses}

Continuous data were expressed as the mean \pm SD or median (range). Statistical analysis was performed using SPSS 13.0 for Windows (SPSS Inc., Chicago, IL, USA). The Kolmogorov-Smirnov test was used to determine the distribution type of the data. The independent sample $t$-test or Bonferroni correction for repeated measurements were used for normally distributed data. The Fisher's exact test and Mann-Whitney $U$-test were used for categorical and skewed data. $P<0.05$ was considered statistically significant.

\section{Results}

A total of 63 patients were recruited for this study; of these patients, 55 completed the study and were included in the final statistical analysis. Three patients were excluded before randomization because of preoperative chronic pain or poor distribution of the epidural block. Another five patients were withdrawn from the study due to changing medical treatments: two required open thoracotomy and three required 
prolonged assisted mechanical ventilation in ICU (Figure 1). Demographic and intraoperative variables were similar between the two groups (Table 1). During the surgery, 30 patients $(54.5 \%)$ were treated with vasoconstrictions; no significant differences existed between the two groups (14 vs $16, P=0.69$; Table 1).

Between 12 and 72 postoperative hours, the cumulative consumptions of fentanyl and ropivacaine were significantly lower in the intermittent group compared with the continuous group at each recorded time point $(P<0.01$; Figure $2 \mathrm{~A}, \mathrm{~B})$. In addition, the total consumption of the two epidural drugs was reduced by more than $30 \%(P=0.001$; Table 2$)$. Patients with continuous epidural infusion received more intravenous fluid during the day of surgery ( $P=0.02$; Table 2$)$. Even though patients with intermittent epidural bolus were associated with reduced ICU stay compared to patients receiving continuous

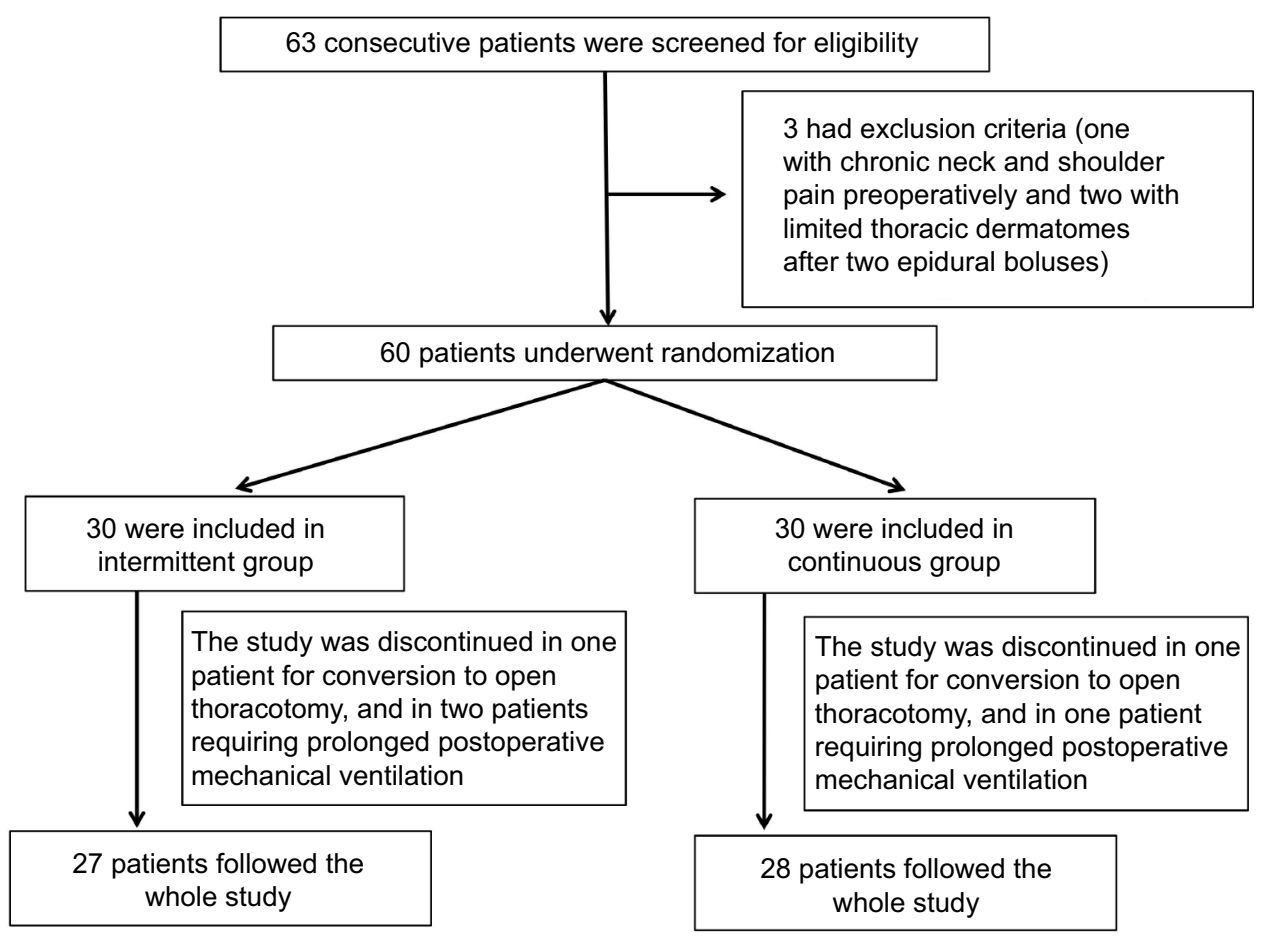

Figure I Flow diagram for inclusion and randomization of patients.

Table I Demographic data and intraoperative variables

\begin{tabular}{|c|c|c|c|}
\hline & I group $(n=27)$ & C group $(n=28)$ & $P$-value \\
\hline Age, years & $61.3 \pm 6.7$ & $63.8 \pm 5.4$ & 0.13 \\
\hline $\mathrm{BMI}, \mathrm{kg} / \mathrm{cm}^{2}$ & $20.5 \pm 4.3$ & $21.4 \pm 3.3$ & 0.25 \\
\hline ASA physical health status, $n(\%)$ & & & 0.62 \\
\hline ASA I & $4(15)$ & $6(21)$ & \\
\hline ASA II & $17(63)$ & $14(50)$ & \\
\hline ASA III & $6(22)$ & $8(29)$ & \\
\hline Smokers/nonsmokers, $\mathrm{n}$ & $17 / 10$ & $15 / 13$ & 0.48 \\
\hline $\operatorname{Sex}(M / F), n$ & $20 / 7$ & $22 / 6$ & 0.69 \\
\hline Duration of surgery, minutes & $255(190,370)$ & $271(185,380)$ & 0.37 \\
\hline Intraoperative blood loss, $\mathrm{mL}$ & $200(100,600)$ & $175(80,450)$ & 0.56 \\
\hline Intraoperative crystalloids volume, $\mathrm{mL}$ & $2,200(1,600,3,300)$ & $2,450(1,700,3,200)$ & 0.28 \\
\hline Intraoperative colloids volume, $\mathrm{mL}$ & $700(500,1,100)$ & $850(500,1,300)$ & 0.31 \\
\hline Number of patients requiring vasoconstrictions, $\mathrm{n}(\%)$ & $14(52)$ & $16(57)$ & 0.69 \\
\hline Intraoperative mean remifentanil dose, $\mathrm{ng} / \mathrm{kg} /$ minute & $86.6 \pm 18.2$ & $78.4 \pm 21.8$ & 0.14 \\
\hline
\end{tabular}

Notes: Continuous data are presented as mean \pm SD or median (range), categorical variables are presented as count (\%). I group, intermittent bolus group; C group, continuous infusion group. $P<0.05$ is considered statistically significant.

Abbreviations: ASA, American Society of Anesthesiologists; BMI, Body mass index; M/F, male/female. 

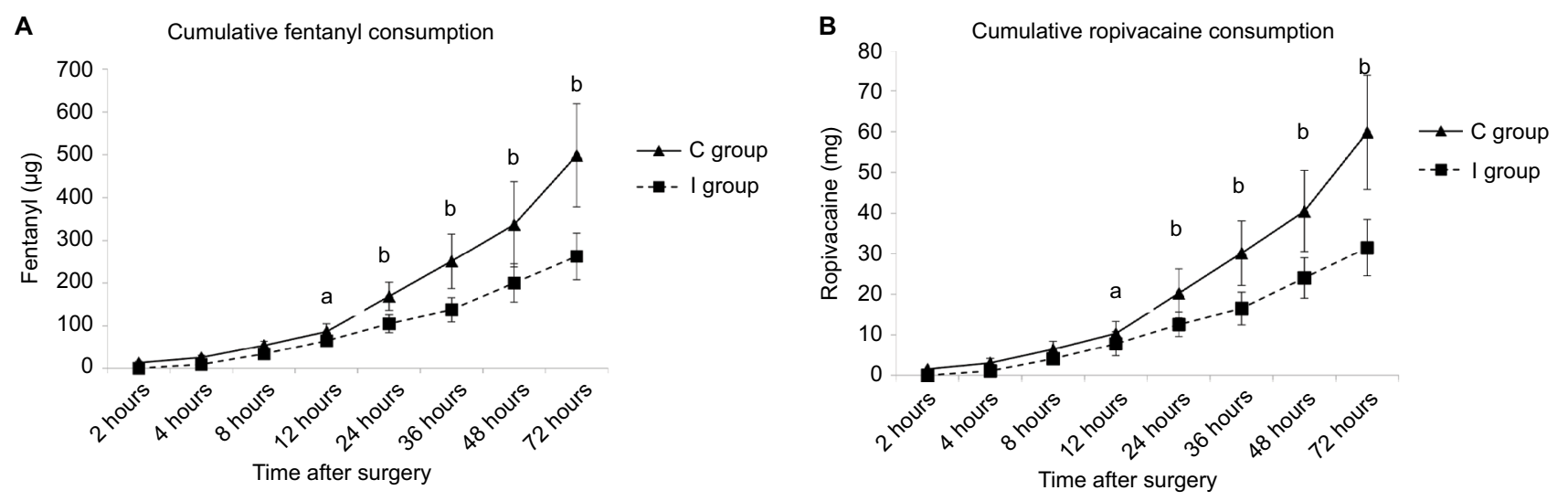

Figure 2 Comparison of ropivacaine and fentanyl consumption after surgery.

Notes: Cumulative fentanyl consumption (A) and ropivacaine consumption (B) were recorded at 2, 4, 8, $12,24,48$, and 72 hours after surgery. Data are presented as the mean \pm SD. I group, intermittent bolus group; $C$ group, continuous infusion group. $A P<0.05$ was considered statistically significant. ${ }^{a}<0.05$ I group vs $C$ group; ${ }^{b} P<0.0$ I I group vs $\mathrm{C}$ group.

Table 2 Postoperative outcome

\begin{tabular}{|c|c|c|c|}
\hline & I group $(n=27)$ & C group $(n=28)$ & $P$-value \\
\hline Time between end of anesthesia and extubation, minutes & $36.7 \pm 15.9$ & $33.4 \pm 13.5$ & 0.41 \\
\hline Total dose requirement of ropivacaine, $\mathrm{mg}$ & $39.4 \pm 12.1$ & $60.3 \pm 11.5$ & 0.001 \\
\hline Total dose requirement of fentanyl, $\mu \mathrm{g}$ & $279.4 \pm 52.6$ & $490.7 \pm 35.3$ & 0.001 \\
\hline Time to first ambulation, hours & $28(20,4 I)$ & $33(19,42)$ & 0.12 \\
\hline Maximal VAS pain scores at rest during PCEA & $2(2,3)$ & $3(2,4)$ & 0.18 \\
\hline Maximal VAS pain scores on coughing during PCEA & $4.5(3,6)$ & $5(3,7)$ & 0.11 \\
\hline Patient satisfaction on PCEA, $\mathrm{n}(\%)$ & & & 0.45 \\
\hline Excellent & $18(66.7)$ & $14(50)$ & \\
\hline Average & $6(22.2)$ & $9(32.1)$ & \\
\hline Poor & $3(I I .1)$ & $5(17.9)$ & \\
\hline Number of patients requiring rescue analgesics during PCEA, $n(\%)$ & $4(14.8)$ & $12(42.9)$ & 0.02 \\
\hline Total amount of intravenous fluid administered in the day of surgery, $\mathrm{mL}$ & $3430.4 \pm 546.2$ & $3763.5 \pm 498.7$ & 0.02 \\
\hline Duration of ICU stay, days & $2.5(1,4)$ & $4(1,7)$ & 0.06 \\
\hline Time for thoracic drainage, days & $8(3,15)$ & $9(4,27)$ & 0.87 \\
\hline Time to hospital discharge, days & $16(9,23)$ & $18(10,4 \mid)$ & 0.10 \\
\hline
\end{tabular}

Notes: Continuous data are presented as mean \pm SD or median (range), categorical variables are presented as count (\%). I group, intermittent bolus group; $C$ group, continuous infusion group. $P<0.05$ is considered statistically significant.

Abbreviations: ICU, intensive care unit; PCEA, patient-controlled epidural analgesia; VAS, visual analog scale.

epidural infusion, the difference between the two groups did not reach statistical significance ( 2.5 vs 4 days, $P=0.06$ ). Similarly, no significant difference was found in the duration of hospital stay ( 16 vs 18 days, $P=0.10$; Table 2).

During the three postoperative days with PCEA, no significant differences existed in the maximal VAS pain score or the median pain score at rest or while coughing (Table 2; Figure 3A, B). At the end of the study, similar patient satisfaction on postoperative analgesia was found between groups $(P=0.45)$ (Table 2). The intermittent group had fewer patients who required additional rescue analgesics for break- through pain during PCEA than the continuous group (4 vs 12, $P=0.02$ ) (Table 2). Ten patients (35.7\%) in the continuous group and two patients (7.4\%) in the intermittent group required repeated doses of additional rescue analgesics $(P=0.01)$ (Figure 4). Fifteen patients (53.6\%) in the continuous group and five patients (18.5\%) in the intermittent group experienced at least one episode of hypotension $(P=0.006)$. No postoperative headache was observed in the two groups. No significant differences were found in the frequency of respiratory depression, postoperative nausea and vomiting, pneumonia, atelectasis, chylothorax, anastomotic leakage, 
A

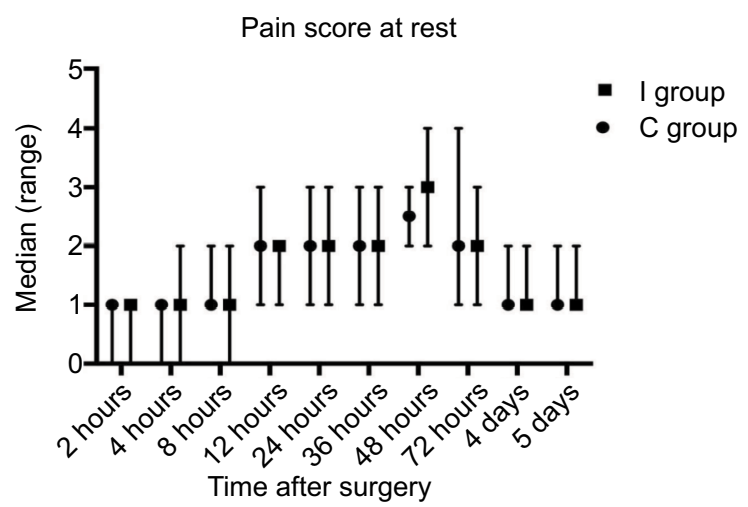

B

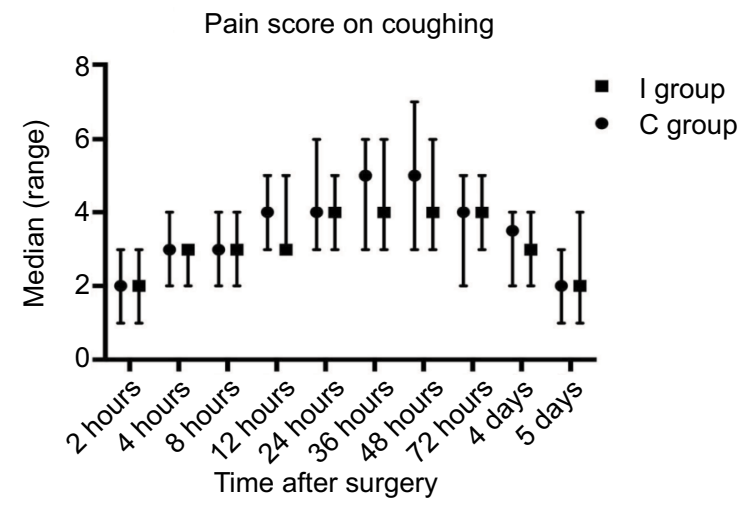

Figure 3 Comparison of pain scores after surgery.

Notes: Intensity of pain was measured at 2, 4, 8, 12, 24, 36, 48, 72 hours, and on the fourth and fifth days after the surgery by VAS at rest (A) or while coughing (B). Data are presented as median (range). I group, intermittent bolus group; $\mathrm{C}$ group, continuous infusion group.

Abbreviation: VAS, visual analog scale.

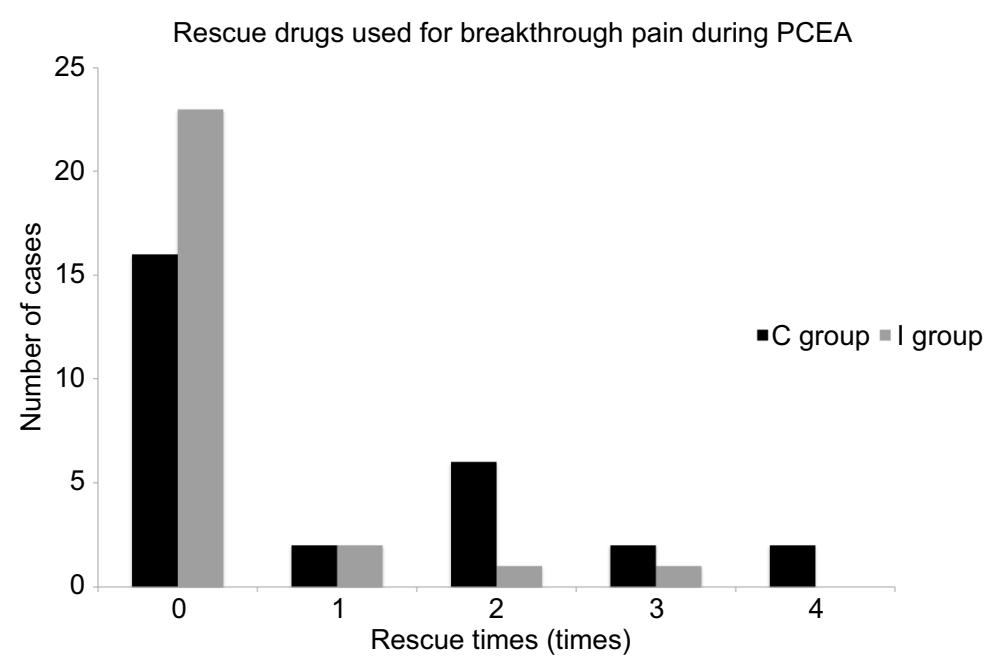

Figure 4 Comparison of the number of patients requiring additional rescue drugs for breakthrough pain.

Notes: I group, intermittent bolus group; $C$ group, continuous infusion group.

Abbreviation: PCEA, patient-controlled epidural analgesia.

atrial arrhythmia, and excessive sedation between the two groups (Table 3 ).

\section{Discussion}

This prospective, randomized study compared the analgesic efficiency and side effects of two dosing regimens in TEA for patients undergoing TLE. In two groups matched by patient characteristics, similar intraoperative treatment and identical epidural solutions, PCEA by intermittent bolus was better than continuous epidural infusion in terms of lower consumption of epidural drugs for comparable pain relief, lower incidence of breakthrough pain, and lower systemic hemodynamic impairment.
According to the dosage regimens of the two groups, the possible doses of epidural drugs administered within an hour were $6,9,12$, and $15 \mathrm{~mL}$ in the continuous group (corresponding to $0,1,2$, and 3 PCEA boluses) vs 0,6 , and $12 \mathrm{~mL}$ (corresponding to 0,1 , and 2 PCEA boluses) in the intermittent group. With smaller daily maximal dosages, the on-demand intermittent epidural bolus resulted in a more than $30 \%$ reduction of the total consumption of ropivacaine and fentanyl to obtain pain relief comparable to that with continuous infusion. In fact, studies on lumbar epidural analgesia have revealed that bolus administration may generate greater injection pressure and facilitate better spread of the anesthetic solution in the epidural space. ${ }^{11,12}$ Direct evidence from the 
Table 3 Postoperative complications

\begin{tabular}{|l|l|l|l|}
\hline Events, $\mathbf{n}$ (\%) & I group $(\mathbf{n = 2 7 )}$ & C group $\mathbf{( n = 2 8 )}$ & P-value \\
\hline Postdural puncture headache & $0(0)$ & $0(0)$ & $>0.99$ \\
Hypotension & $5(18.5)$ & $15(53.6)$ & 0.006 \\
Respiratory depression & $0(0)$ & $2(7.1)$ & 0.15 \\
Pneumonia & $5(18.5)$ & $5(17.9)$ & 0.95 \\
Atelectasis & $4(14.8)$ & $4(14.3)$ & 0.96 \\
Anastomotic leakage & $3(11.1)$ & $5(17.9)$ & 0.48 \\
Chylothorax & I (3.7) & $1(3.6)$ & 0.98 \\
Nausea and vomit & $3(11.1)$ & $5(17.9)$ & 0.48 \\
Atrial arrhythmia & I (3.7) & $2(7.1)$ & 0.53 \\
\hline
\end{tabular}

Notes: Categorical variables are presented as count (\%). I group, intermittent bolus group; $C$ group, continuous infusion group. $P<0.05$ is considered statistically significant.

cadaveric dissection with freezing microtome sectioning also showed a more uniform spread of the epidural solution when large volumes and high injection pressure were used..$^{13}$ To our knowledge, the current study is the first to confirm the high efficiency of intermittent epidural injections in TEA for pain management after TLE.

Another improvement of intermittent bolus in TEA is its ability to achieve more hemodynamic stability. Postoperative hypotension following esophagectomy was reported in a wide range of patients (between $17.5 \%$ and $76 \%$ ) who received TEA. ${ }^{14-16}$ Because different volumes and concentrations of local anesthetics were used in these studies, it is difficult to compare the values directly. The incidence of hypotension in the present study ( $18.5 \%$ by intermittent bolus vs $53.6 \%$ by continuous infusion) was included in this range, with many more episodes of hypotension recorded in patients with continuous epidural infusion. One possible explanation for this discrepancy in hemodynamic depression between the two TEA regimens is that the maintenance of analgesia with continuous epidural infusion results in the need for rescue boluses to treat breakthrough pain when low infusion rates are used or in more episodes of hypotension when higher infusion rates are used (in an attempt to decrease the need for rescue boluses). Conversely, when epidural bolus was administered by patients on demand instead of at scheduled or fixed time intervals, the accumulation of local anesthetics and extensive epidural block was avoided. Another cause should be attributed to a high efficiency of the bolus injection. Compared with continuous infusion, a much smaller dose of local anesthetics was required by epidural bolus to produce an identical sensory blockade of the necessary dermatomes.

No significant differences in other postoperative complications were observed between the two groups, and equal levels of patient satisfaction on postoperative analgesia were achieved. This study also showed similar incidence of anastomotic leakage following the two epidural regimens, even though more frequent hypotension was induced by continuous epidural infusion. TEA has been observed to have a paradoxical effect on blood flow to stomach anastomosis. Some studies attributed the reduction of anastomotic leakage to vasodilation following sympathetic blockade, which was supported by evidence from human and animal studies that improved perfusion in gastric conduit during TEA. ${ }^{17,18}$ In contrast, severe hypotension is also a potential risk to anastomotic leak or gastric tube necrosis due to regional ischemia. ${ }^{19}$ It seems that this negative impact on anastomotic blood flow could be effectively prevented if normal hemodynamics were maintained. In a previous study on esophagectomy, a reversal of the TEA-induced low flux was observed at the anastomotic end of the newly formed gastric tube by intravenous phenylephrine infusion. ${ }^{20}$ Our results did not show any association between the incidence of hypotension and anastomotic leakage. Additional fluids and vasoconstrictors were used for early correction of hypotension found in this study. On the day of surgery, patients with continuous epidural infusion accepted a larger amount of fluid infusion.

Multimodal analgesia consisting of TEA and systemic NSAIDs were used in all patients. Epidural analgesia was initiated before operation to relieve intraoperative stress hormone responses and avoid amplified sensory processing, both of which were essential for faster postoperative recovery and better results. A progressively increased pain was observed after TLE, and pain intensity peaked during 36-48 hours after surgery. This time was a little earlier than reported in the literature on open esophagectomy, in which the highest pain score during mobilization reported occurred on the second or third day. ${ }^{21,22} \mathrm{~A}$ decreased pain scale was observed on the fourth postoperative day, and pain while 
coughing could be controlled below a score of 4 in most of the patients by intravenous NSAIDs alone.

\section{Study limitations}

There were several limitations in our study. First, the analgesic efficiency of the two TEA regimens was compared in patients undergoing TLE instead of open esophagectomy. It is well known that the gold standard of TEA is primarily based on open esophagectomy, whereas in minimally invasive surgery, paravertebral or transverse abdominis plane block has become increasingly popularity. However, as one of the most commonly used postoperative analgesic techniques, TEA has been shown to have a few advantages in both open and minimally invasive esophagectomy. ${ }^{23}$ Current results proposed a preferable delivery mode for TEA when it is utilized in TLE. A comprehensive comparison between TEA and peripheral nerve blockades on postoperative pain control, side effects, and rehabilitation is required to determine the optimal analgesic modality for TLE. Second, the difference in the analgesic effect between the two regimens may have been underestimated due to the relatively small sample size in the present study. Our results did not show any difference in pain score at rest or while coughing between the two groups. Nevertheless, decreased breakthrough pain was observed when TEA was administered by intermittent bolus, which indicates the potential that a high efficacy of pain relief existed in this delivery mode. Lastly, although it did not reach statistical significance, a trend of shorter ICU stay was observed in the patients with intermittent epidural bolus. This improvement on postoperative rehabilitation was thought to be related to a lower use of epidural narcotics, less postoperative fluid loading, and more stable hemodynamics. However, more convincing data are required to confirm the benefit of this epidural technology on postoperative outcomes after TLE.

\section{Conclusion}

TEA by on-demand intermittent bolus is a more efficient and safer alternative to continuous epidural infusion for pain management after TLE. This method may provide comparable pain relief with greatly reduced consumption of local anesthetics and opioids and lead to lower hemodynamic impairment.

\section{Acknowledgments}

The authors thank Prof Ming Du and Huanwen Chen from the Department of Thoraco-Cardiac Surgery of The First Affiliated Hospital of Chongqing Medical University for their assistance in this study and helpful comments. The present work was supported by grants from the Chongqing Science and
Technology Commission (project no. cstc2015jcsf10008) and Chongqing Health Commission (project no. 2017HBRC001).

\section{Disclosure}

The authors report no conflicts of interest in this work.

\section{References}

1. Raymond DP, Seder CW, Wright CD, et al. Predictors of major morbidity or mortality after resection for esophageal cancer: a society of thoracic surgeons general thoracic surgery database risk adjustment model. Ann Thorac Surg. 2016;102(1):207-214.

2. Lv L, Hu W, Ren Y, Wei X. Minimally invasive esophagectomy versus open esophagectomy for esophageal cancer: a meta-analysis. Onco Targets Ther. 2016;9:6751-6762.

3. Straatman J, van der Wielen N, Cuesta MA, et al. Minimally invasive versus open esophageal resection: three-year follow-up of the previously reported randomized controlled trial: the TIME trial. Ann Surg. 2017;266(2):232-236.

4. Weiss R, Pöpping DM. Is epidural analgesia still a viable option for enhanced recovery after abdominal surgery. Curr Opin Anaesthesiol. 2018;31(5):1-629.

5. Feltracco P, Bortolato A, Barbieri S, et al. Perioperative benefit and outcome of thoracic epidural in esophageal surgery: a clinical review. Dis Esophagus. 2018;31(5).

6. Durkin C, Schisler T, Lohser J. Current trends in anesthesia for esophagectomy. Curr Opin Anaesthesiol. 2017;30(1):30-35.

7. Findlay JM, Gillies RS, Millo J, Sgromo B, Marshall RE, Maynard ND. Enhanced recovery for esophagectomy: a systematic review and evidence-based guidelines. Ann Surg. 2014;259(3):413-431.

8. Gourlay GK, Plummer JL, Cherry DA, et al. Comparison of intermittent bolus with continuous infusion of epidural morphine in the treatment of severe cancer pain. Pain. 1991;47(2):135-140.

9. Salim R, Nachum Z, Moscovici R, Lavee M, Shalev E. Continuous compared with intermittent epidural infusion on progress of labor and patient satisfaction. Obstet Gynecol. 2005;106(2):301-306.

10. Singh S, Singh A, Srivastava U. Low dose epidural analgesia during labor: comparison between patient controlled epidural analgesia with basal continuous infusion and intermittent bolus technique. $J$ Obstet Gynaecol India. 2011;61(4):404-407.

11. Fettes PD, Moore CS, Whiteside JB, McLeod GA, Wildsmith JA. Intermittent vs continuous administration of epidural ropivacaine with fentanyl for analgesia during labour. Br JAnaesth. 2006;97(3):359-364.

12. Carvalho B, George RB, Cobb B, McKenzie C, Riley ET. Implementation of programmed intermittent epidural bolus for the maintenance of labor analgesia. Anesth Analg. 2016;123(4):965-971.

13. Hogan Q. Distribution of solution in the epidural space: examination by cryomicrotome section. Reg Anesth Pain Med. 2002;27(2):150-156.

14. Brown MJ, Kor DJ, Allen MS, et al. Dual-epidural catheter technique and perioperative outcomes after Ivor-Lewis esophagectomy. Reg Anesth Pain Med. 2013;38(1):3-8.

15. Levy G, Cordes MA, Farivar AS, Aye RW, Louie BE. Transversus abdominis plane block improves perioperative outcome after esophagectomy versus epidural. Ann Thorac Surg. 2018;105(2):406-412.

16. Niwa Y, Koike M, Torii K, et al. Combination of continuous paravertebral block and epidural anesthesia in postoperative pain control after esophagectomy. Esophagus. 2016;13(1):42-47.

17. Lázár G, Kaszaki J, Abrahám S, et al. Thoracic epidural anesthesia improves the gastric microcirculation during experimental gastric tube formation. Surgery. 2003;134(5):799-805.

18. Michelet P, Roch A, D'Journo XB, et al. Effect of thoracic epidural analgesia on gastric blood flow after oesophagectomy. Acta Anaesthesiol Scand. 2007;51(5):587-594.

19. Al-Rawi OY, Pennefather SH, Page RD, Dave I, Russell GN. The effect of thoracic epidural bupivacaine and an intravenous adrenaline infusion on gastric tube blood flow during esophagectomy. Anesth Analg. 2008;106(3):884-887. 
20. Pathak D, Pennefather SH, Russell GN, et al. Phenylephrine infusion improves blood flow to the stomach during oesophagectomy in the presence of a thoracic epidural analgesia. Eur J Cardiothorac Surg. 2013;44(1):130-133.

21. Flisberg P, Törnebrandt K, Walther B, Lundberg J. Pain relief after esophagectomy: thoracic epidural analgesia is better than parenteral opioids. J Cardiothorac Vasc Anesth. 2001;15(3):282-287.
22. Rudin A, Flisberg P, Johansson J, Walther B, Lundberg CJ. Thoracic epidural analgesia or intravenous morphine analgesia after thoracoabdominal esophagectomy: a prospective follow-up of 201 patients. $J$ Cardiothorac Vasc Anesth. 2005;19(3):350-357.

23. Zingg U, McQuinn A, Divalentino D, et al. Minimally invasive versus open esophagectomy for patients with esophageal cancer. Ann Thorac Surg. 2009;87(3):911-919.

\section{Publish your work in this journal}

The Journal of Pain Research is an international, peer reviewed, open access, online journal that welcomes laboratory and clinical findings in the fields of pain research and the prevention and management of pain. Original research, reviews, symposium reports, hypothesis formation and commentaries are all considered for publication.

\section{Dovepress}

The manuscript management system is completely online and includes a very quick and fair peer-review system, which is all easy to use. Visit http://www.dovepress.com/testimonials.php to read real quotes from published authors. 